\title{
A versatile pathway to end- functionalized cellulose ethers for click chemistry applications
}

\section{$\operatorname{AUTHOR}(\mathrm{S}):$}

Kamitakahara, Hiroshi; Suhara, Ryo; Yamagami, Mao; Kawano, Haruko; Okanishi, Ryoko; Asahi, Tomoyuki; Takano, Toshiyuki

\section{CITATION:}

Kamitakahara, Hiroshi ...[et al]. A versatile pathway to end-functionalized cellulose ethers for click chemistry applications. Carbohydrate Polymers 2016, 151: 88-95

\section{ISSUE DATE:}

2016-10-20

URL:

http://hdl.handle.net/2433/217323

\section{RIGHT:}

(c) 2016. This manuscript version is made available under the CC-BY-NC-ND 4 . license

http://creativecommons.org/licenses/by-nc-nd/4.0/; The full-text file will be made open to the public on 20 October 2017 in accordance with publisher's 'Terms and Conditions for Self-Archiving'.; This is not the published version. Please cite only the published version.; この論文は出版社版でありません。引用の際には出版社版をご確認ご利用ください。 
1 A versatile pathway to end-functionalized cellulose ethers for click chemistry 2 applications

3

4 Hiroshi Kamitakahara*, Ryo Suhara, Mao Yamagami, Haruko Kawano, Ryoko Okanishi, Tomoyuki

5 Asahi, Toshiyuki Takano

6 Graduate School of Agriculture, Kyoto University, Sakyo-ku, Kyoto 606-8502, Japan

$7 \quad{ }^{*}$ Corresponding author: hkamitan@kais.kyoto-u.ac.jp

8 Phone: +81-75-753-6257

9 Fax: +81-75-753-6300

10

11 


\section{ABSTRACT $^{1}$}

This paper describes a versatile pathway to heterobifunctional/telechelic cellulose ethers, such as tri-O-methyl cellulosyl azide and propargyl tri-O-methyl celluloside, having one free C-4 hydroxyl group attached to the glucosyl residue at the non-reducing end for the use in Huisgen 1,3-dipolar cycloaddition and copper(I)-catalyzed azide-alkyne cycloaddition (CuAAC). The one-step end-functionalization of cellulose ethers for molecular rod synthesis involves the introduction of two reactive groups at both ends of the cellulose molecule, and can afford linear triblock copolymers via CuAAC and further reactions. We were able to tailor the degree of polymerization of end-functionalized cellulose ethers with controlled amounts of a Lewis acid, namely $\mathrm{SnCl}_{4}$. Chemical structures of the above cellulose ethers and the reaction conditions for controlling molecular length are discussed.

Keywords: end-functionalized cellulose ether; copper(I)-catalyzed azide-alkyne cycloaddition; functional molecular rod; tri-O-methyl cellulosyl azide; propargyl tri-O-methyl celluloside

\section{Introduction}

Methylcellulose (MC), being one of the more common cellulose ethers, has been of interest in the investigation of structure-property relationships, such as thermoreversible gelation properties at elevated temperature. Our research focuses on the design and synthesis of regioselectively methylated cellulose derivatives via ring-opening polymerization of glucose orthopivalate derivatives (Kamitakahara, Hori, \& and Nakatsubo, 1996; Karakawa, Mikawa, Kamitakahara, \& Nakatsubo, 2002; Nakatsubo, Kamitakahara, \& Hori, 1996) or from natural cellulose (Kamitakahara, Koschella, Mikawa, Nakatsubo, Heinze, \& Klemm, 2008; Nakagawa et al., 2012) and diblock methylcellulose with regioselective functionalization patterns (Nakagawa, Fenn, Koschella, Heinze, \& Kamitakahara, 2011a, b; Nakagawa, Steiniger, Richter, Koschella, Heinze, \& Kamitakahara, 2012). As a result, we found that a diblock structure composed of hydrophilic cellobiosyl and hydrophobic 2,3,6-tri-O-methylcellulosyl segments is crucial for the thermoreversible gelation of aqueous MC solutions (Nakagawa, Fenn, Koschella, Heinze, Kamitakahara, 2011a). Commercial MC prepared under heterogeneous conditions is an alternating block copolymer composed of densely substituted hydrophobic and less densely substituted hydrophilic block sequences (Savage, 1957). The synthetic route to multiblock MC derivatives composed of hydrophobic 2,3,6-tri-O-methylcellulosyl and hydrophilic cellulosyl segments remains open.

\footnotetext{
1 Copper(I)-catalyzed azide-alkyne cycloaddition, CuAAC; methylcellulose, MC; cellulose triacetate, CTA; gel permeation chromatography, GPC; 2,5-dihydroxybenzoic acid, DHB; degree of polymerization, DP.
} 
Precise control of the monosaccharide sequence to prepare multiblock derivatives is, however, extremely difficult and time-consuming. We synthesized 1,2,3-triazole-linked diblock MC composed of low molecular weight cellulose and 2,3,6-tri-O-methyl cellulose (Nakagawa, Kamitakahara, \& Takano, 2012) and found that a 2 wt. \% aqueous solution of this MC analogue exhibited thermoreversible gelation behavior, meaning that linkages between hydrophilic and hydrophobic segments do not affect gelation properties. Thus, we considered utilizing linkages other than the glycosidic bond to prepare multiblock MC copolymers.

To be suitable building blocks for the multiblock MC copolymers, the cellulose derivatives must have functional groups at both ends of the linear molecule. Heterobifunctional/telechelic derivatives are more desirable than homobifunctional/telechelic ones (Kim, Stannett, \& Gilbert, 1973, 1976; Pohjola \& Eklund, 1977; Steinmann, 1968, 1970) for the preparation of multiblock copolymers. Derivatives having two different functional groups at both ends of the linear polymer are therefore attractive and promising for the exploration of a new research field in cellulose chemistry.

62 On the other hand, cellulose derivatives are known to be semi-rigid polymers (De Oliveira \& 63 Glasser, 1994), which controls their physical properties. The concept of a 'molecular rod' is 64 therefore applicable to heterobifunctional/telechelic cellulose derivatives, which can be viewed as 65 bricks of a molecular Lego (Lepage, Schneider, Bodlenner, \& Compain, 2015; Meldal, 2008). To 66 connect several bricks of the cellulosic Lego, two ends of the molecular rod must be separately functionalized under independent activation conditions. It is then possible to covalently bind several molecular bricks, adding other brick units under different reaction conditions.

We have previously reported the synthesis of tri-O-acetyl cellulosyl azide (Kamitakahara, Enomoto, Hasegawa, \& Nakatsubo, 2005). This molecule is a key compound for the end-functionalization of cellulose derivatives. The azide group can be easily converted into an amino group, which can be used in a subsequent amidation reaction. For instance, we successfully synthesized cellulose triacetate (CTA)-block-oligoamide-15 (Kamitakahara, Enomoto, Hasegawa, \& Nakatsubo, 2005; Kamitakahara \& Nakatsubo, 2005), a CTA derivative carrying a single pyrene group at the reducing end (Enomoto, Kamitakahara, Takano, \& Nakatsubo, 2006), and a CTA derivative having a single lipoic acid moiety at the reducing end (Enomoto-Rogers, Kamitakahara, Yoshinaga, \& Takano, 2010). The high reactivity of the azide group towards alkynes is known as the click chemistry approach, and is based on Huisgen 1,3-dipolar cycloaddition and copper(I)-catalyzed azide-alkyne cycloaddition (CuAAC) (Kolb, Finn, \& Sharpless, 2001). We have prepared comb-shaped graft 
81 copolymers with CTA side chains (Enomoto-Rogers, Kamitakahara, Yoshinaga, \& Takano, 2012) and CTA-block-poly( $\gamma$-benzyl-L-glutamate) (Kamitakahara, Baba, Yoshinaga, Suhara, \& Takano, 2014), knowing that the CuAAC reaction is a more powerful tool for bonding two polymeric segments than amidation.

Not only cellulose esters, such as cellulose acetate, but also a representative cellulose ether, methylcellulose, were also important molecular Lego bricks. Methyl tri-O-methyl celluloside, with a single hydroxyl group at the C-4 position of the glucosyl residue at the non-reducing end was prepared by methanolysis of 2,3,6-tri-O-methyl cellulose (Nakagawa, Fenn, Koschella, Heinze, \& Kamitakahara, 2011b; Nakagawa, Kamitakahara, \& Takano, 2011). Propargylation of one end of the cellulose ether derivative afforded a cellulose ether carrying a single alkyne group at the end of the cellulosic molecular rod, methyl tri-O-methyl celluloside (Nakagawa, Kamitakahara, \& Takano, 2012). Cellulose ethers are more stable than the corresponding esters in both acidic and alkaline reaction conditions used to construct the cellulosic molecular architecture. Thus, we focused on the synthesis of cellulosic molecular rods carrying two independent end-functional groups.

Propargylated methyl tri-O-methyl celluloside was synthesized from commercial methylcellulose in three reaction steps: complete methylation, methanolysis, and propargylation. This molecular rod has a functional group at one end (Nakagawa, Fenn, Koschella, Heinze, \& Kamitakahara, 2011b), which is a disadvantage. Therefore, we were motivated to synthesize cellulosic molecular rods carrying two independent end-functional groups, in other words, cellulosic heterobifunctional/telechelic polymers.

To introduce an azide group at the C-1 position of the glucosyl residue at the reducing end of CTA, it was treated with hydrogen bromide in acetic acid to afford the $\alpha$-anomer of tri- $O$-acetyl cellulosyl bromide. The bromide was then treated with acetic acid and silver oxide to yield the $\beta$-anomer of acetyl tri-O-acetyl cellulose, which was finally converted into the $\beta$-anomer of tri-O-acetyl cellulosyl azide using trimethylsilyl azide and $\mathrm{SnCl}_{4}$ (Kamitakahara, Enomoto, Hasegawa, \& Nakatsubo, 2005). We tried to produce tri-O-methyl cellulosyl azide (2) with a controlled molecular weight from tri-O-methyl cellulose (1) in a one-step reaction. Azide and alkyne groups form a pair for the 1,3-dipolar cycloaddition, and preparing propargyl tri-O-methyl celluloside (3) is, therefore, of critical importance. Thus, we attempted to produce propargyl tri-O-methyl celluloside (3) with a controlled molecular weight from tri-O-methyl cellulose (1) in a one-step reaction.

Moreover, the free C-4 hydroxyl of the glucosyl residue at the non-reducing end could connect with other molecular bricks having epoxide, acyl, isocyanate, and other functionalities, thereby 
117 extending the variety of molecular architecture motifs. Heterobifunctional/telechelic cellulose 118 derivatives, at least, provide molecules with triblock structures. The production of cellulosic 119 triblock copolymers from homobifunctional/telechelic cellulose derivatives has already been 120 reported (Kim, Stannett, \& Gilbert, 1973, 1976; Pohjola \& Eklund, 1977; Steinmann, 1968, 1970), 121 however, heterobifunctional/telechelic cellulose derivatives are still unknown, to the best of our 122 knowledge.

124 Consequently, the aim of this research was to find the appropriate reaction conditions affording 125 end-functionalized cellulose ethers, such as tri-O-methyl cellulosyl azide (2) and propargyl 126 tri-O-methyl celluloside (3), for click chemistry and further conversion using the remaining 127 functionalized end of the ethers. This paper describes well-controlled synthetic methods for 128 preparing cellulosic precursors for CuAAC, namely tri-O-methyl cellulosyl azide (2) and propargyl 129 tri-O-methyl celluloside (3). The reaction conditions used to introduce azide and propargyl groups 130 onto the tri-O-methyl cellulose (1) scaffold and the structures of reaction products are also 131 discussed.

\section{MATERIALS AND METHODS}

\section{$134 \quad$ 2.1. Materials}

135 All reagents and solvents were obtained from Nacalai Tesque, Wako Chemical, and Sasaki 136 Chemical, Japan, and were used as received.

\subsection{Analytical measurements}

$139{ }^{1} \mathrm{H}$ and ${ }^{13} \mathrm{C}$ NMR spectra were acquired in $\mathrm{CDCl}_{3}$ on a Varian $500 \mathrm{NMR}$ spectrometer at room temperature. The molecular weights of the products were measured by gel permeation chromatography (GPC) in chloroform on a Shimadzu SEC system (CBM-20A, SPD-10AvP, SIL-10A, LC-10ATVP, FCV-10ALvp, CTO-10Avp, RID-10A, and FRC-10A, Shimadzu, Japan). Sample solutions were passed through a syringe filter (Sartorius Stedim, Minisart RC 4 or RC 15; pore size $0.45 \mu \mathrm{m}$ ) before GPC analysis. Shodex columns (K802, K802.5, and K805) with a guard column (Shodex, K-G) were used. Number- and weight-averaged molecular weights $\left(M_{\mathrm{n}}\right.$ and $\left.M_{\mathrm{w}}\right)$ and polydispersity indices $\left(M_{\mathrm{w}} / M_{\mathrm{n}}\right)$ were estimated using polystyrene standards (Shodex). Matrix-assisted laser desorption/ionization time-of-flight mass spectra (MALDI-TOF MS) were recorded on a Bruker Autoflex III machine in the positive ion linear mode. 2,5-Dihydroxybenzoic acid (DHB) was used as a matrix for these measurements.

\subsection{Synthetic methods}

\subsubsection{2,3,6-Tri-O-methyl cellulose (1)}


153 Complete methylation of SM-400 (Shin-Etsu Chemical, Japan) to afford 2,3,6-tri-O-methyl 154 cellulose (1) was carried out as previously described (Nakagawa, Kamitakahara, \& Takano, 2011).

155 MALDI-TOF MS (positive linear mode; DHB matrix):

156 DP (degree of polymerization) $=5: \mathrm{C}_{47} \mathrm{H}_{86} \mathrm{O}_{26}$ Calcd. $[\mathrm{M}]^{+}$1066.54; Found $[\mathrm{M}+\mathrm{Na}]^{+}=1089.637$

157 DP = 6: $\mathrm{C}_{56} \mathrm{H}_{102} \mathrm{O}_{31}$ Calcd. $[\mathrm{M}]^{+}$1270.64; Found $[\mathrm{M}+\mathrm{Na}]^{+}=1293.733$

158 DP = 7: $\mathrm{C}_{65} \mathrm{H}_{118} \mathrm{O}_{36}$ Calcd. $[\mathrm{M}]^{+}$1474.74; Found $[\mathrm{M}+\mathrm{Na}]^{+}=1497.964$

159 DP = 8: $\mathrm{C}_{74} \mathrm{H}_{134} \mathrm{O}_{41}$ Calcd. [M] $]^{+}$1678.84; Found $[\mathrm{M}+\mathrm{Na}]^{+}=1701.954$

$160 \mathrm{DP}=9: \mathrm{C}_{83} \mathrm{H}_{150} \mathrm{O}_{46}$ Calcd. $[\mathrm{M}]^{+}$1882.94; Found $[\mathrm{M}+\mathrm{Na}]^{+}=1905.979$

161 DP = 10: $\mathrm{C}_{92} \mathrm{H}_{166} \mathrm{O}_{51}$ Calcd. [M] ${ }^{+}$2087.04; Found $[\mathrm{M}+\mathrm{Na}]^{+}=2109.946$

$162 \mathrm{DP}=11: \mathrm{C}_{101} \mathrm{H}_{182} \mathrm{O}_{56}$ Calcd. $[\mathrm{M}]^{+}$2291.14; Found $[\mathrm{M}+\mathrm{Na}]^{+}=2313.76$

$163 \mathrm{DP}=12: \mathrm{C}_{110} \mathrm{H}_{198} \mathrm{O}_{61}$ Calcd. $[\mathrm{M}]^{+}$2495.24; Found $[\mathrm{M}+\mathrm{Na}]^{+}=2517.776$

164 DP = 13: $\mathrm{C}_{119} \mathrm{H}_{214} \mathrm{O}_{66}$ Calcd. [M] 2699.34; Found $[\mathrm{M}+\mathrm{Na}]^{+}=2721.576$

$165 \mathrm{DP}=14: \mathrm{C}_{128} \mathrm{H}_{230} \mathrm{O}_{71}$ Calcd. $[\mathrm{M}]^{+}$2903.44; Found $[\mathrm{M}+\mathrm{Na}]^{+}=2926.146$

$166 \mathrm{DP}=15: \mathrm{C}_{137} \mathrm{H}_{246} \mathrm{O}_{76}$ Calcd. $[\mathrm{M}]^{+}$3107.54; Found $[\mathrm{M}+\mathrm{Na}]^{+}=3129.431$

167 DP = 16: $\mathrm{C}_{146} \mathrm{H}_{262} \mathrm{O}_{81}$ Calcd. [M] $]^{+}$3311.64; Found $[\mathrm{M}+\mathrm{Na}]^{+}=3332.968$

168 DP $=17: \mathrm{C}_{155} \mathrm{H}_{278} \mathrm{O}_{86}$ Calcd. $[\mathrm{M}]^{+}$3515.74; Found $[\mathrm{M}+\mathrm{Na}]^{+}=3536.968$

$169 \mathrm{DP}=18: \mathrm{C}_{164} \mathrm{H}_{294} \mathrm{O}_{91}$ Calcd. $[\mathrm{M}]^{+}$3717.84; Found $[\mathrm{M}+\mathrm{Na}]^{+}=3740.437$

170 DP = 19: $\mathrm{C}_{173} \mathrm{H}_{310} \mathrm{O}_{96}$ Calcd. $[\mathrm{M}]^{+}$3923.94; Found $[\mathrm{M}+\mathrm{Na}]^{+}=3944.958$

\subsubsection{Tri-O-methyl cellulosyl azide (2)}

173 To a solution of tri-O-methyl cellulose (1) $\left(51.1 \mathrm{mg}, M_{\mathrm{n}}=2.58 \times 10^{-4}, D P_{\mathrm{n}}=126\right)$ in anhydrous 174 chloroform $(0.633 \mathrm{~mL})$ were added $0.32 \mathrm{~mL}$ of trimethylsilyl azide $(0.2 \mathrm{~mL})$ in anhydrous 175 chloroform (9.8 mL) (TMS- $\mathrm{N}_{3}: 4.87 \times 10^{-2} \mathrm{mmol}, 0.195$ equiv./anhydro glucose unit (AGU)) and $1760.047 \mathrm{~mL}$ of tin(IV) tetrachloride $(0.1 \mathrm{~mL})$ in anhydrous chloroform $(4.9 \mathrm{~mL})\left(\mathrm{SnCl}_{4}: 8.16 \times 10^{-3}\right.$ mmol, 0.034 equiv./AGU). The reaction mixture was stirred at room temperature (r.t.) for $4 \mathrm{~h}$ and was subsequently neutralized with $0.113 \mathrm{~mL}$ of triethylamine $(0.2 \mathrm{~mL})$ in chloroform $(9.8 \mathrm{~mL})$ $\left(\mathrm{Et}_{3} \mathrm{~N}\right.$ : 2 equiv. with respect to $\left.\mathrm{SnCl}_{4}\right)$. The reaction mixture was extracted with ethyl acetate, washed with water and brine, dried over $\mathrm{Na}_{2} \mathrm{SO}_{4}$, and concentrated in vacuo to give tri-O-methyl cellulosyl azide (2) (42.6 mg).

${ }^{1} \mathrm{H}-\mathrm{NMR}$ (500 MHz, $\mathrm{CDCl}_{3}$ ): $\delta 2.96$ (t, $\left.J=9.0 \mathrm{~Hz}, \mathrm{H} 2\right), 3.22$ (t, $J=9.0 \mathrm{~Hz}, \mathrm{H} 3$ ), 3.29 (broad d, $J=$ $9.0 \mathrm{~Hz}, \mathrm{H} 5), 3.39\left(\mathrm{OCH}_{3}\right), 3.54\left(\mathrm{OCH}_{3}\right), 3.58\left(\mathrm{OCH}_{3}\right), 3.62-3.84(\mathrm{H} 4, \mathrm{H} 6), 4.28(\mathrm{~d}, J=8.0, \mathrm{H} 1)$, 4.34 (d, $J=8.0$, internal H1), 4.47 (d, $J=8.5 \mathrm{~Hz}, \mathrm{H} 1-\beta$ at reducing end), 5.45 (d, $J=3.5 \mathrm{~Hz}, \mathrm{H1}-\alpha$ at reducing end).

${ }^{13} \mathrm{C}-\mathrm{NMR}\left(125 \mathrm{MHz}, \mathrm{CDCl}_{3}\right): \delta$ 59.0, 59.1, $59.1\left(\mathrm{C} 6-\mathrm{OCH}_{3}\right), 59.2,59.4,60.1,60.3\left(\mathrm{C} 3-\mathrm{OCH}_{3}\right)$, 
189 (C2 internal), 84.6, 84.9, 85.0 (C3 internal), 85.1, 87.0 (C1 $\alpha$ at reducing end), 87.3, 89.9 (C1 $\beta$ at 190 reducing end), 103.1 (C1 internal), 103.3, 103.4.

191 MALDI-TOF MS (positive linear mode; DHB matrix):

$192 \mathrm{DP}=5: \mathrm{C}_{45} \mathrm{H}_{81} \mathrm{~N}_{3} \mathrm{O}_{25}$ Calcd. $[\mathrm{M}]^{+}$1063.52; Found $[\mathrm{M}+\mathrm{Na}]^{+}=1086.441$

$193 \mathrm{DP}=6: \mathrm{C}_{54} \mathrm{H}_{97} \mathrm{~N}_{3} \mathrm{O}_{30}$ Calcd. $[\mathrm{M}]^{+}$1267.62; Found $[\mathrm{M}+\mathrm{Na}]^{+}=1290.637$

194 DP = 7: $\mathrm{C}_{63} \mathrm{H}_{113} \mathrm{~N}_{3} \mathrm{O}_{35}$ Calcd. $[\mathrm{M}]^{+}$1471.72; Found $[\mathrm{M}+\mathrm{Na}]^{+}=1494.81$

$195 \mathrm{DP}=8: \mathrm{C}_{72} \mathrm{H}_{129} \mathrm{~N}_{3} \mathrm{O}_{40}$ Calcd. [M] $]^{+}$1675.82; Found $[\mathrm{M}+\mathrm{Na}]^{+}=1698.909$

$196 \mathrm{DP}=9$ : $\mathrm{C}_{81} \mathrm{H}_{145} \mathrm{~N}_{3} \mathrm{O}_{45}$ Calcd. $[\mathrm{M}]^{+}$1879.92; Found $[\mathrm{M}+\mathrm{Na}]^{+}=1902.891$

197 DP = 10: $\mathrm{C}_{90} \mathrm{H}_{161} \mathrm{~N}_{3} \mathrm{O}_{50}$ Calcd. $[\mathrm{M}]^{+}$2084.01; Found $\left[\mathrm{M}-\mathrm{N}_{2}+\mathrm{Na}\right]^{+}=2078.970,\left[\mathrm{M}-\mathrm{N}_{2}+\mathrm{K}\right]^{+}=$ 198 2094.96, $[\mathrm{M}+\mathrm{Na}]^{+}=2106.909,[\mathrm{M}+\mathrm{K}]^{+}=2122.97$

199 DP = 11: $\mathrm{C}_{99} \mathrm{H}_{177} \mathrm{~N}_{3} \mathrm{O}_{55}$ Calcd. $[\mathrm{M}]^{+}$2288.11; Found $[\mathrm{M}+\mathrm{Na}]^{+}=2310.949$

$200 \mathrm{DP}=12: \mathrm{C}_{108} \mathrm{H}_{193} \mathrm{~N}_{3} \mathrm{O}_{60}$ Calcd. $[\mathrm{M}]^{+}$2492.21; Found $[\mathrm{M}+\mathrm{Na}]^{+}=2515.075$

201 DP = 13: $\mathrm{C}_{117} \mathrm{H}_{209} \mathrm{~N}_{3} \mathrm{O}_{65}$ Calcd. [M] $]^{+}$2696.31; Found $[\mathrm{M}+\mathrm{Na}]^{+}=2718.929$

202 DP = 14: $\mathrm{C}_{126} \mathrm{H}_{225} \mathrm{~N}_{3} \mathrm{O}_{70}$ Calcd. $[\mathrm{M}]^{+}$2900.41; Found $[\mathrm{M}+\mathrm{Na}]^{+}=2922.819$

$203 \mathrm{DP}=15: \mathrm{C}_{135} \mathrm{H}_{241} \mathrm{~N}_{3} \mathrm{O}_{75}$ Calcd. $[\mathrm{M}]^{+}$3104.51; Found $[\mathrm{M}+\mathrm{Na}]^{+}=3126.248$

$204 \mathrm{DP}=16: \mathrm{C}_{144} \mathrm{H}_{257} \mathrm{~N}_{3} \mathrm{O}_{80}$ Calcd. $[\mathrm{M}]^{+}$3308.61; Found $[\mathrm{M}+\mathrm{Na}]^{+}=3331.014$

205 DP = 17: $\mathrm{C}_{153} \mathrm{H}_{273} \mathrm{~N}_{3} \mathrm{O}_{85}$ Calcd. $[\mathrm{M}]^{+} 3512.71$; Found $[\mathrm{M}+\mathrm{Na}]^{+}=3534.132$

$206 \mathrm{DP}=18: \mathrm{C}_{162} \mathrm{H}_{289} \mathrm{~N}_{3} \mathrm{O}_{90}$ Calcd. $[\mathrm{M}]^{+}$3716.81; Found $[\mathrm{M}+\mathrm{Na}]^{+}=3738.273$

207 DP = 19: $\mathrm{C}_{171} \mathrm{H}_{305} \mathrm{~N}_{3} \mathrm{O}_{95}$ Calcd. $[\mathrm{M}]^{+}$3920.91; Found $[\mathrm{M}+\mathrm{Na}]^{+}=3942.31$.

208

\subsubsection{Propargyl tri- $O$-methyl celluloside (3)}

210 To a solution of tri-O-methyl cellulose (1) (50 mg, $\left.M_{\mathrm{n}}=2.58 \times 10^{-4}, D P_{\mathrm{n}}=126\right)$ in anhydrous 211 dichloromethane ( $1 \mathrm{~mL}$ ) were added 2-propyne-1-ol ( $4.2 \mu \mathrm{L}, 7.1 \times 10^{-2} \mathrm{mmol}, 0.3$ equiv./AGU) and $212 \mathrm{SnCl}_{4}(2.4 \mu \mathrm{L}, 0.021 \mathrm{mmol}, 0.085$ equiv./AGU). The reaction mixture was stirred at r.t. for $4 \mathrm{~h}$ 213 and was subsequently extracted with chloroform, washed with water and brine, dried over $\mathrm{Na}_{2} \mathrm{SO}_{4}$, 214 and concentrated in vacuo to give propargyl tri-O-methyl celluloside (3) (42 mg).

$215{ }^{1} \mathrm{H}-\mathrm{NMR}\left(500 \mathrm{MHz}, \mathrm{CDCl}_{3}\right): \delta 2.43\left(\mathrm{CH}_{2} \mathrm{CC} \underline{\mathrm{H}}\right), 2.95$ (t, $\left.J=9.0 \mathrm{~Hz}, \mathrm{H} 2\right), 3.20$ (t, $J=9.0 \mathrm{~Hz}, \mathrm{H} 3$ ) 2163.28 (broad d, $J=9.0 \mathrm{~Hz}, \mathrm{H} 5), 3.37\left(\mathrm{OCH}_{3}\right), 3.53\left(\mathrm{OCH}_{3}\right), 3.57\left(\mathrm{OC}_{3}\right)$, 3.62-3.66 (H6), 3.72-3.81 217 (H6), 3.69 (t, $J=9.0 \mathrm{~Hz}, \mathrm{H} 4), 4.28-4.29$ ( $\left.\underline{\mathrm{C}}_{2} \mathrm{CCH}\right), 4.34$ (d, 1H, $J=8.0$, internal H1), 4.38-4.39 $218\left(\mathrm{CH}_{2} \mathrm{CCH}\right), 4.49$ (d, $J=8.0 \mathrm{~Hz}, \mathrm{H} 1-\beta$ at reducing end), 5.20 (d, $J=4.0 \mathrm{~Hz}, \mathrm{H} 1-\alpha$ at reducing end). $219{ }^{13} \mathrm{C}-\mathrm{NMR}\left(125 \mathrm{MHz}, \mathrm{CDCl}_{3}\right): \delta 54.4\left(\underline{\mathrm{CH}}_{2} \mathrm{CCH}(\mathrm{C} 1-\alpha)\right)$, $55.6\left(\underline{\mathrm{CH}}_{2} \mathrm{CCH}(\mathrm{C} 1-\beta)\right)$, 58.4, 59.0, 59.1 220 (C6-O $\left.\underline{C H}_{3}\right), 59.2,59.3,59.6,60.1,60.3\left(\mathrm{C} 3-\underline{O C H}_{3}\right), 60.3,60.4,60.5\left(\mathrm{C} 2-\underline{O C H}_{3}\right), 60.7,60.8,70.0$, 22170.2 (C6 (internal)), 70.4, 72.0, 73.1, 73.3, 74.6, 74.7, 74.8 (C5 internal), 74.9, 77.1, 77.2, 77.4 (C4 222 internal), 77.5, 77.6, 77.9, 78.7, 78.9, 79.3, 80.7, 81.0, 82.8 (C2 at reducing end (C1- $\beta$ )), 83.3, 83.5 223 (C2 internal), 83.7, 84.4 (C3 at reducing end (C1- $\beta$ )), 84.9. 85.0 (C3 internal), 86.1, 94.5 (C1- $\alpha$ at 224 reducing end), 100.7 (C1- $\beta$ at reducing end), 101.2, 103.1 (C1 internal), 103.2, 103.3. 
225

226

227

228

229

230

231

232

233

234

235

236

237

238

239

240

241

242

243

244

245

246

247

248

249

250

MALDI-TOF MS (positive linear mode; DHB as matrix):

$\mathrm{DP}=4: \mathrm{C}_{39} \mathrm{H}_{68} \mathrm{O}_{21}$ Calcd. $[\mathrm{M}]^{+}$872.43; Found $[\mathrm{M}+\mathrm{Na}]^{+}=895.164$

$\mathrm{DP}=5: \mathrm{C}_{48} \mathrm{H}_{84} \mathrm{O}_{26}$ Calcd. $[\mathrm{M}]^{+}$1076.53; Found $[\mathrm{M}+\mathrm{Na}]^{+}=1099.249$

$\mathrm{DP}=6: \mathrm{C}_{57} \mathrm{H}_{100} \mathrm{O}_{31}$ Calcd. $[\mathrm{M}]^{+}$1280.62; Found $[\mathrm{M}+\mathrm{Na}]^{+}=1303.425$

$\mathrm{DP}=7: \mathrm{C}_{66} \mathrm{H}_{116} \mathrm{O}_{36}$ Calcd. $[\mathrm{M}]^{+}$1484.72; Found $[\mathrm{M}+\mathrm{Na}]^{+}=1507.497$

$\mathrm{DP}=8: \mathrm{C}_{75} \mathrm{H}_{132} \mathrm{O}_{41}$ Calcd. $[\mathrm{M}]^{+}$1688.82; Found $[\mathrm{M}+\mathrm{Na}]^{+}=1711.584$

$\mathrm{DP}=9: \mathrm{C}_{84} \mathrm{H}_{148} \mathrm{O}_{46}$ Calcd. $[\mathrm{M}]^{+}$1892.92; Found $[\mathrm{M}+\mathrm{Na}]^{+}=1915.701$

$\mathrm{DP}=10: \mathrm{C}_{93} \mathrm{H}_{164} \mathrm{O}_{51}$ Calcd. $[\mathrm{M}]^{+}$2097.02; Found $[\mathrm{M}+\mathrm{Na}]^{+}=2119.702$

$\mathrm{DP}=11: \mathrm{C}_{102} \mathrm{H}_{180} \mathrm{O}_{56}$ Calcd. $[\mathrm{M}]^{+}$2301.12; Found $[\mathrm{M}+\mathrm{Na}]^{+}=2323.797$

$\mathrm{DP}=12: \mathrm{C}_{111} \mathrm{H}_{196} \mathrm{O}_{61}$ Calcd. $[\mathrm{M}]^{+}$2505.22; Found $[\mathrm{M}+\mathrm{Na}]^{+}=2527.612$

$\mathrm{DP}=13: \mathrm{C}_{120} \mathrm{H}_{212} \mathrm{O}_{66}$ Calcd. $[\mathrm{M}]^{+}$2709.32; Found $[\mathrm{M}+\mathrm{Na}]^{+}=2731.001$

$\mathrm{DP}=14: \mathrm{C}_{129} \mathrm{H}_{228} \mathrm{O}_{71}$ Calcd. $[\mathrm{M}]^{+}$2913.42; Found $[\mathrm{M}+\mathrm{Na}]^{+}=2936.027$

$\mathrm{DP}=15: \mathrm{C}_{138} \mathrm{H}_{244} \mathrm{O}_{76}$ Calcd. $[\mathrm{M}]^{+}$3117.52; Found $[\mathrm{M}+\mathrm{Na}]^{+}=3139.696$

$\mathrm{DP}=16: \mathrm{C}_{147} \mathrm{H}_{260} \mathrm{O}_{81}$ Calcd. $[\mathrm{M}]^{+}$3321.62; Found $[\mathrm{M}+\mathrm{Na}]^{+}=3343.723$

$\mathrm{DP}=17: \mathrm{C}_{156} \mathrm{H}_{276} \mathrm{O}_{86}$ Calcd. $[\mathrm{M}]^{+}$3525.72; Found $[\mathrm{M}+\mathrm{Na}]^{+}=3547.69$

$\mathrm{DP}=18: \mathrm{C}_{165} \mathrm{H}_{292} \mathrm{O}_{91}$ Calcd. $[\mathrm{M}]^{+}$3729.82; Found $[\mathrm{M}+\mathrm{Na}]^{+}=3752.824$

$\mathrm{DP}=19: \mathrm{C}_{174} \mathrm{H}_{308} \mathrm{O} 96$ Calcd. $[\mathrm{M}]^{+}$3933.92; Found $[\mathrm{M}+\mathrm{Na}]^{+}=3956.686$.

\section{Results and Discussion}

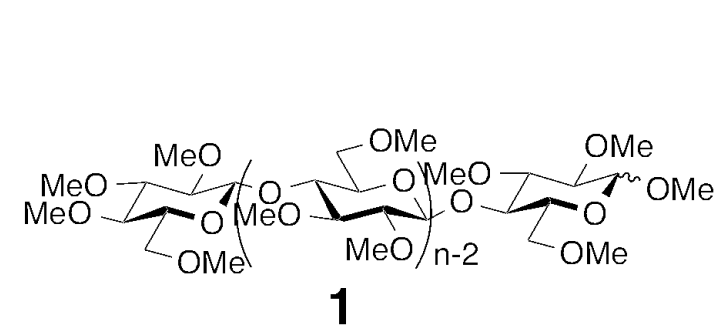

1

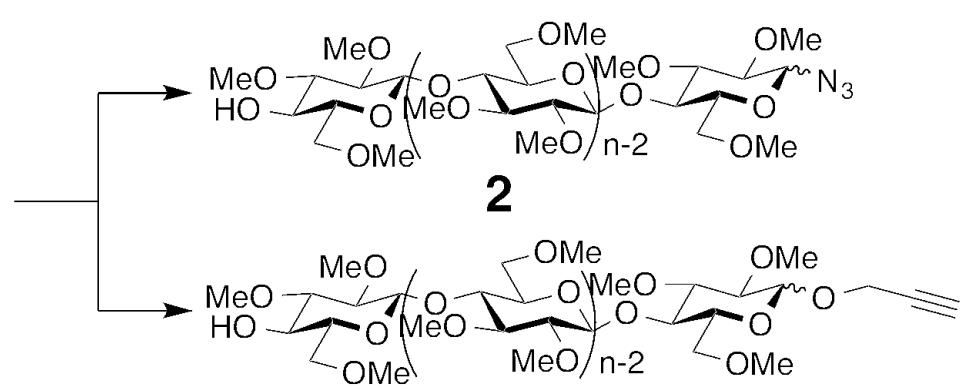

3

Scheme 1. Synthesis of tri-O-methyl cellulosyl azide (2) and propargyl tri-O-methyl celluloside (3) from tri-O-methyl cellulose (1).

\subsection{Tri-O-methyl cellulose (1)}

The MALDI-TOF MS spectrum of tri-O-methyl cellulose (1) (Figure 1A) indicates that the base peak (among peaks with the same DP) corresponds to the pseudo molecular ion $[\mathrm{M}+\mathrm{Na}]^{+}$of the fully methylated methylcellulose with methyl groups at both ends of the molecule, meaning that both the C-1 hydroxyl of the glucosyl residue at the reducing end and the C-4 hydroxyl of the glucosyl residue at the non-reducing end are methylated. The spectrum, however, also showed peaks with lower intensities. For instance, sodium adduct ion peaks were found, corresponding to 
255

256

257

258

tri-O-methyl cellulose (1) with a few non-methylated hydroxyl groups on the cellulosic backbone.

Pseudo molecular ion $[\mathrm{M}+\mathrm{Na}]^{+}$peaks with $\mathrm{m} / \mathrm{z}=2313.760$ and 2299.870 were attributed to completely methylated methylcellulose $(\mathrm{DP}=11)$ and to methylcellulose $(\mathrm{DP}=11)$ with one hydroxyl group, respectively.

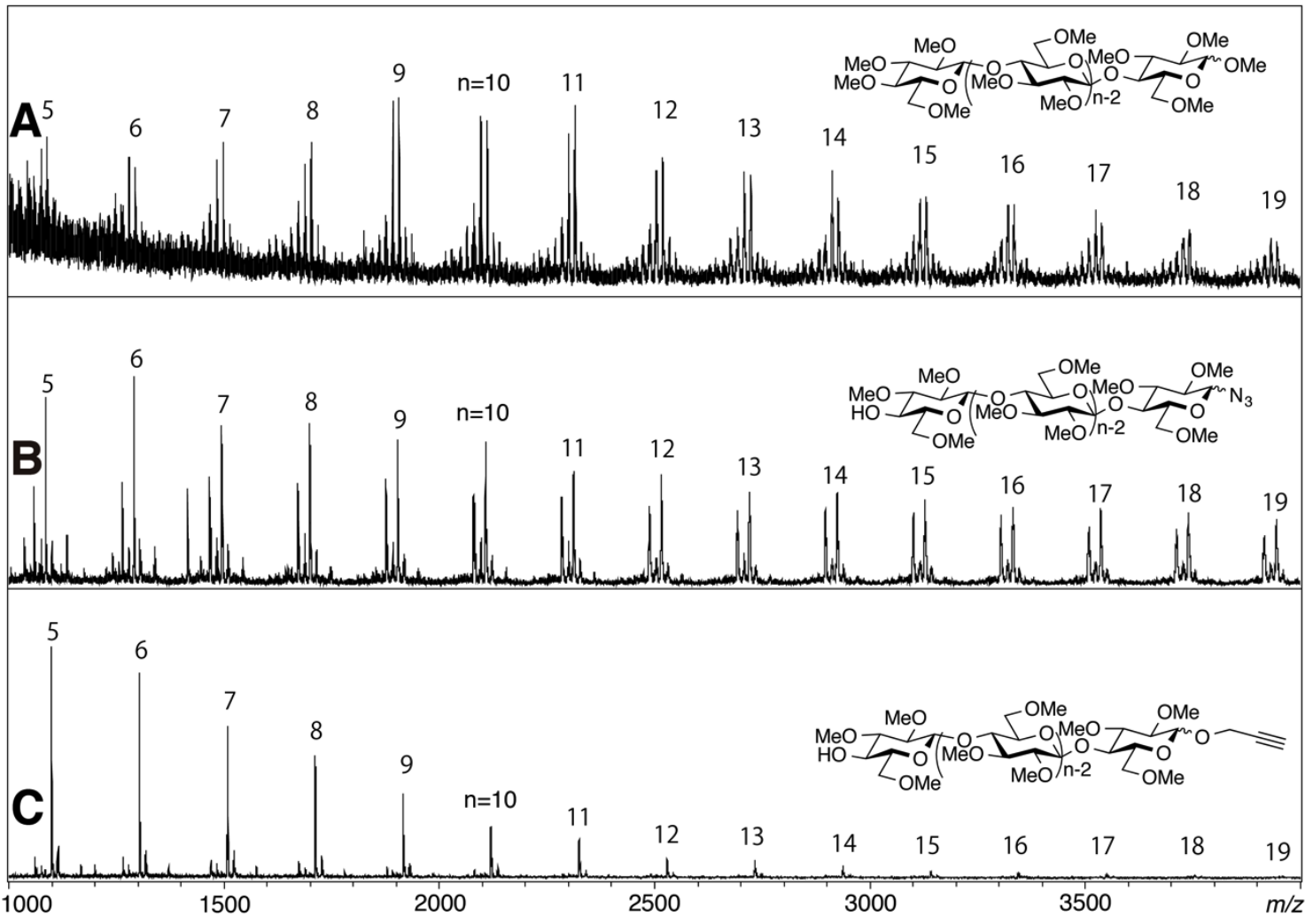

Figure 1. MALDI-TOF MS spectra of (A) tri-O-methyl cellulose (1) $\left(D P_{\mathrm{n}}=322\right)$, (B) tri-O-methyl cellulosyl azide (2) $\left(D P_{\mathrm{n}}=27.5\right)$, and $(\mathrm{C})$ propargyl tri-O-methyl celluloside (3) $\left(D P_{\mathrm{n}}=13.2\right)$.

\subsection{Synthesis of tri-O-methyl cellulosyl azide (2)}

We tried to prepare the tri-O-methyl cellulosyl azide (2) using the synthetic strategy used for tri-O-acetyl- $\beta$-cellulosyl azide (Kamitakahara, Enomoto, Hasegawa, \& Nakatsubo, 2005). We have already found that the $\alpha$-anomer of acetyl tri-O-acetyl cellulose is relatively stable under azidation conditions using trimethylsilyl azide and $\mathrm{SnCl}_{4}$. To replace the anomeric acetyl group with azide, a mixture of acetate $\alpha$-and $\beta$-anomers was first converted to the $\beta$-anomer of acetyl tri- $O$-acetyl cellulose via an $\mathrm{S}_{\mathrm{N}} 2$ reaction of tri- $O$-acetyl cellulosyl $\alpha$-bromide. The $\beta$-anomer of acetyl tri-O-acetyl cellulose was converted to tri-O-methyl $\beta$-cellulosyl azide. Hydrogen bromide, however, led to intensive degradation of tri-O-methyl cellulose (1), which was more reactive than tri-O-acetyl cellulose.

Due to the higher reactivity of tri-O-methyl cellulose (1) compared to tri-O-acetyl cellulose, we subsequently tried to prepare a mixture of $\alpha$ - and $\beta$-anomers of tri-O-methyl cellulosyl azide (2) from $\alpha$ - and $\beta$-anomers of methyl tri-O-methyl cellulose (1) in a one-step reaction. After the 
optimization of reaction conditions, the above synthesis was successfully accomplished.

278

279

280

281

282

283

284

285

286

287

288

289

290

291

292

293

The MALDI-TOF MS spectrum of tri-O-methyl cellulosyl azide (2) (Figure 1B) shows that it has one free hydroxyl group at the non-reducing end, specifically at the C-4 position of the glucosyl residue. The reaction mechanism to synthesis tri-O-methyl cellulosyl azide (2) having one free C-4 hydroxyl group attached to the glucosyl residue at the non-reducing end from tri-O-methyl cellulose (1) is illustrated in Scheme S1. Repetitive signals consisting of two major peaks are shown. For instance, pseudo molecular ion peaks with $\mathrm{m} / \mathrm{z}=2310.949\left([\mathrm{M}+\mathrm{Na}]^{+}\right)$and $\mathrm{m} / \mathrm{z}=$ $2282.861\left(\left[\mathrm{M}-\mathrm{N}_{2}+\mathrm{Na}\right]^{+}\right)$are attributed to tri-O-methyl cellulosyl azide (2) with $\mathrm{DP}=11$ and one free hydroxyl group attached to the C-4 carbon of the glucosyl residue at the non-reducing end.

Figure 2 shows ${ }^{1} \mathrm{H}-\mathrm{NMR}$ spectra of tri-O-methyl cellulose (1), tri-O-methyl cellulosyl azide (2), and propargyl tri-O-methyl celluloside (3). All proton resonances of tri-O-methyl cellulose (1) were assigned based on previous studies (Karakawa, Mikawa, Kamitakahara, \& Nakatsubo, 2002; Nakagawa, Fenn, Koschella, Heinze, \& Kamitakahara, 2011b). Resonances with low intensities at 5.45 and $4.47 \mathrm{ppm}$ were attributed to $\alpha$ - and $\beta$-anomeric protons of the glucosyl residue at the reducing end of tri-O-methyl cellulosyl azide (2), respectively, as shown in Figure 2B.

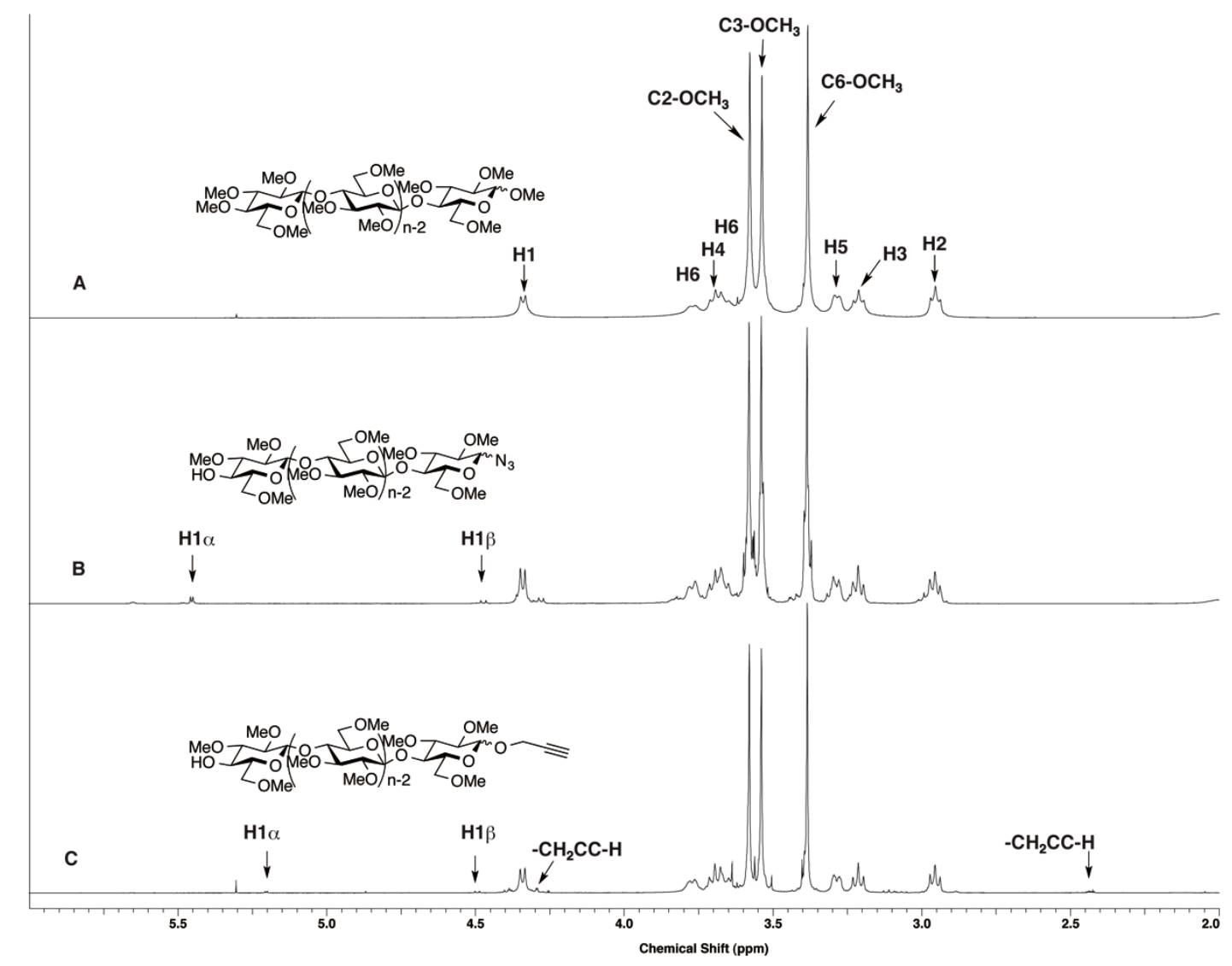

Figure 2. ${ }^{1} \mathrm{H}-\mathrm{NMR}$ spectra of (A) tri-O-methyl cellulose (1) $\left(D P_{\mathrm{n}}=322\right)$, (B) tri-O-methyl cellulosyl azide (2) $\left(D P_{\mathrm{n}}=27.5\right)$, and $(\mathrm{C})$ propargyl tri-O-methyl celluloside $(\mathbf{3})\left(D P_{\mathrm{n}}=71.2\right)$. 
All carbon resonances of tri-O-methyl cellulose (1) were also assigned, as shown in Figure 3. In $\beta$-anomeric carbons of the glucosyl residue at the reducing end of tri-O-methyl cellulosyl azide (2), respectively.

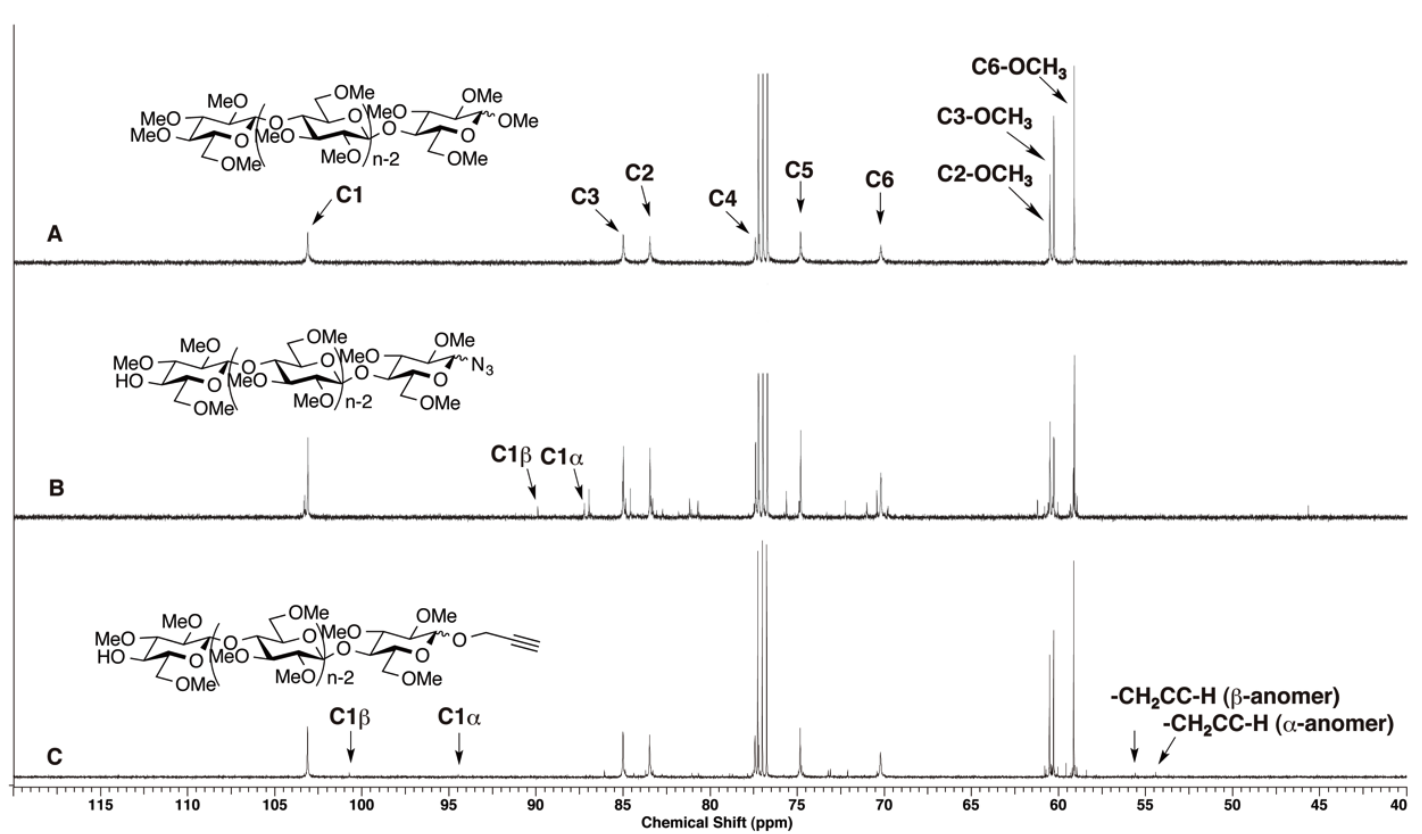

Figure 3. ${ }^{13} \mathrm{C}-\mathrm{NMR}$ spectra of $(\mathrm{A})$ tri-O-methyl cellulose $(\mathbf{1})\left(D P_{\mathrm{n}}=322\right)$, (B) tri-O-methyl cellulosyl azide (2) $\left(D P_{\mathrm{n}}=27.5\right)$, and $(\mathrm{C})$ propargyl tri-O-methyl celluloside $(\mathbf{3})\left(D P_{\mathrm{n}}=71.2\right)$.

\subsection{Synthesis of propargyl tri-O-methyl celluloside (3)}

It was subsequently planned to prepare propargyl tri-O-methyl celluloside (3) from tri-O-methyl cellulose (1) via in a one-step reaction. Propargyl alcohol was coupled at the C-1 position of the glucosyl residue at the reducing end of tri-O-methyl cellulose (1).

The MALDI-TOF MS spectrum of propargyl tri-O-methyl celluloside (3) (Figure 1C) indicates that it has one free C-4 hydroxyl group attached to the glucosyl residue at the non-reducing end $\left(D P_{\mathrm{n}}=\right.$ 13.2, obtained from tri-O-methyl cellulose having $\left.D P_{\mathrm{n}}=65.4\right)$, as exemplified by the detection of a pseudo molecular ion peak $\left([\mathrm{M}+\mathrm{Na}]^{+}\right)$with $\mathrm{m} / \mathrm{z}=2323.797$. The expanded MALDI-TOF MS spectra are shown in Figure S1. The reaction mechanism to synthesis propargyl tri-O-methyl celluloside (3) having one free C-4 hydroxyl group attached to the glucosyl residue at the non-reducing end from tri-O-methyl cellulose (1) is illustrated in Scheme S1.

As shown in Figure 2C, the ${ }^{1} \mathrm{H}-\mathrm{NMR}$ spectrum of propargyl tri-O-methyl celluloside (3) showed signals of alkyne and methylene protons of the propargyl group at 2.43 and $4.28-4.39 \mathrm{ppm}$, 
321

322

323

324

325

326

327

328

329

330

331

332

333

334

335

336

Table 1. Azido end-functionalization of tri-O-methyl cellulose (1).

\begin{tabular}{|c|c|c|c|c|c|}
\hline entry & $\begin{array}{l}\text { TMS-N }_{3} \\
\text { (equiv/AGU) }\end{array}$ & $\begin{array}{l}\mathrm{SnCl}_{4} \\
\text { (equiv./AGU) }\end{array}$ & $M_{\mathrm{n}} / 10^{4}$ & $M_{\mathrm{w}} / M_{\mathrm{n}}$ & $D P_{\mathrm{n}}$ \\
\hline 1 & 0.195 & 0.034 & 1.7 & 2.0 & 81 \\
\hline 2 & 0.390 & 0.067 & 1.1 & 1.9 & 54 \\
\hline 3 & 0.585 & 0.100 & 0.41 & 2.9 & 20 \\
\hline
\end{tabular}

Moreover, end-propargylation of tri-O-methyl cellulose (1) was carried out with 2-propyne-1-ol and

respectively. In the corresponding ${ }^{13} \mathrm{C}-\mathrm{NMR}$ spectrum (Figure 3C), the methylene carbons of the propargyl group appeared at $54.4\left(\underline{\mathrm{CH}}_{2} \mathrm{CCH}(\mathrm{C} 1-\alpha)\right)$ and $55.6 \mathrm{ppm}\left(\underline{\mathrm{CH}}_{2} \mathrm{CCH}(\mathrm{C} 1-\beta)\right)$. Anomeric protons of the glucosyl residue at the reducing end of propargyl tri-O-methyl celluloside (3) appeared at $4.49(\mathrm{H} 1-\beta)$ and $5.20 \mathrm{ppm}(\mathrm{H} 1-\alpha)$, as shown in Figure 2C. In addition, anomeric carbons of the glucosyl residue at the reducing end of propargyl tri-O-methyl celluloside (3) appeared at $94.5(\mathrm{C} 1-\alpha)$ and $100.7 \mathrm{ppm}(\mathrm{C} 1-\beta)$, as shown in Figure 3C.

\subsection{Control of molecular weight of cellulose ethers carrying two independent end-functional} groups

The molecular weight of end-functionalized cellulose ethers influences the physical properties of block copolymers when they are used as one of the molecular Lego bricks. For instance, a well-defined diblock copolymer exhibits microphase separation (Kamitakahara, Baba, Yoshinaga, Suhara, \& Takano, 2014), which has received considerable attention. Molecular lengths of the two segments usually affect microphase separation patterns of diblock copolymers, motivating us to explore reaction conditions for obtaining end-functionalized cellulose ethers with tailored molecular weights.

End-azidation of tri-O-methyl cellulose (1) was carried out with trimethylsilyl azide and $\mathrm{SnCl}_{4}$ in anhydrous chloroform, with reaction conditions summarized in Table 1 . The degree of polymerization of tri-O-methyl cellulosyl azide (2) decreased with increasing amounts of $\mathrm{SnCl}_{4}$. This result means that we are able to control the DP of tri-O-methyl cellulosyl azide (2). Actually, tri-O-methyl cellulosyl azide (2) having one free C-4 hydroxyl group attached to the glucosyl residue at the non-reducing end with DP from 20 to 81 was produced.

$\mathrm{SnCl}_{4}$ in anhydrous dichloromethane, with reaction conditions summarized in Table 2. The DP of propargyl tri-O-methyl celluloside (3) decreased with increasing amounts of $\mathrm{SnCl}_{4}$. This result means that we are also able to control the DP of propargyl tri-O-methyl celluloside (3) having one free C-4 hydroxyl group attached to the glucosyl residue at the non-reducing end, producing the above compound with DP from 29 to 45. 
354 Table 2. Propargyl end-functionalization of tri-O-methyl cellulose (1).

\begin{tabular}{|c|c|c|c|c|c|}
\hline entry & $\begin{array}{l}\text { 2-propyn-1-ol } \\
\text { (equiv./AGU) }\end{array}$ & $\begin{array}{l}\mathrm{SnCl}_{4} \\
\text { (equiv./AGU) }\end{array}$ & $M_{n} / 10^{3}$ & $M_{\mathrm{w}} / M_{\mathrm{n}}$ & $D P_{\mathrm{n}}$ \\
\hline 1 & 0.3 & 0.070 & 9.2 & 1.9 & 45 \\
\hline 2 & 0.3 & 0.085 & 7.7 & 2.2 & 38 \\
\hline 3 & 0.3 & 0.100 & 6.0 & 2.0 & 29 \\
\hline
\end{tabular}

\section{Conclusion}

MALDI-TOF MS, ${ }^{1} \mathrm{H}$ - and ${ }^{13} \mathrm{C}-\mathrm{NMR}$ spectra confirm that the end-functionalization of fully methylated cellulose proceeded to afford cellulosic molecular rods carrying two independent end groups at the both ends of the molecules. Controlled degradation of the fully protected cellulose ethers by Lewis acid in presence of trimethylsilyl azide or propargyl alcohol produced tri-O-methyl cellulosyl azide (2) and propargyl tri-O-methyl celluloside (3) with tailored DP, respectively, having a free hydroxyl group at the C-4 position of the non-reducing glucopyranosyl residue. These methods furnished end-functionalized cellulose ethers as semi-rigid linear hydrophobic molecular Lego bricks with tunable degrees of polymerization. The free C-4 hydroxyl of the glucosyl residue at the non-reducing end could connect with other molecular bricks, thereby extending the variety of molecular architecture motifs. The developed chemistry will enable us to initiate a new era of precise block architecture of polysaccharide derivatives.

\section{Acknowledgements}

We thank the Japan Society for the Promotion of Science (JSPS) for their financial support of this study, in part through Grant-in-Aid for Scientific Research (Nos. 24380092 and 15H04531).

\section{Supporting Information}

375 Reaction mechanisms of the tri-O-methyl cellulosyl azide (2) and propargyl tri-O-methyl celluloside (3) syntheses from tri-O-methyl cellulose (1) are illustrated in Scheme S1. MALDI-TOF MS spectra of tri-O-methyl cellulose (1), tri-O-methyl cellulosyl azide (2), and propargyl tri-O-methyl celluloside (3) in the region of $D P=11$ are shown in Figure S1. MALDI-TOF MS spectra of tri-O-methyl cellulose (1) with a $D P_{\mathrm{n}}$ of 322, obtained using positive ion linear mode, are shown in Figure S2. Expanded ${ }^{1} \mathrm{H}$ - and ${ }^{13} \mathrm{C}-\mathrm{NMR}$ spectra of tri-O-methyl cellulose (1) $\left(D P_{\mathrm{n}}=322\right)$, tri-O-methyl cellulosyl azide (2) $\left(D P_{\mathrm{n}}=27.5\right)$, and propargyl tri-O-methyl celluloside $(3)\left(D P_{\mathrm{n}}=71.2\right)$ in the anomeric proton region are shown in Figures S3 and S4, respectively. 


\section{References}

De Oliveira, W., \& Glasser, W. G. (1994). Novel cellulose derivatives. II. Synthesis and characteristics of mono-functional cellulose propionate segments. Cellulose, 1, 77-86.

Enomoto, Y., Kamitakahara, H., Takano, T., \& Nakatsubo, F. (2006). Synthesis of diblock copolymers with cellulose derivatives. 3. Cellulose derivatives carrying a single pyrene group at the reducing-end and fluorescent studies of their self-assembly systems in aqueous $\mathrm{NaOH}$ solutions. Cellulose, 13(4), 437-448.

Enomoto-Rogers, Y., Kamitakahara, H., Yoshinaga, A., \& Takano, T. (2010). Radially oriented cellulose triacetate chains on gold nanoparticles. Cellulose, 17(5), 923-936.

Enomoto-Rogers, Y., Kamitakahara, H., Yoshinaga, A., \& Takano, T. (2012). Comb-shaped graft copolymers with cellulose side-chains prepared via click chemistry. Carbohydrate Polymers, 87(3), 2237-2245.

Kamitakahara, H., Baba, A., Yoshinaga, A., Suhara, R., \& Takano, T. (2014). Synthesis and crystallization-induced microphase separation of cellulose triacetate-block-poly( $\gamma$-benzyl-L-glutamate). Cellulose, 21, 3323-3338.

Kamitakahara, H., Enomoto, Y., Hasegawa, C., \& Nakatsubo, F. (2005). Synthesis of diblock copolymers with cellulose derivatives. 2. Characterization and thermal properties of cellulose triacetate-block-oligoamide-15. Cellulose, 12(5), 527-541.

Kamitakahara, H., Hori, M., \& Nakatsubo, F. (1996). Substituent effect on ring-opening polymerization of regioselectively acylated $\alpha$-D-glucopyranose 1,2,4-orthopivalate derivatives. Macromolecules, 29(19), 6126-6131.

Kamitakahara, H., Koschella, A., Mikawa, Y., Nakatsubo, F., Heinze, T., \& Klemm, D. (2008). Syntheses and comparison of 2,6-di-O-methyl celluloses from natural and synthetic celluloses. Macromolecular Bioscience, 8(7), 690-700.

Kamitakahara, H., \& Nakatsubo, F. (2005). Synthesis of diblock copolymers with cellulose derivatives. 1. Model study with azidoalkyl carboxylic acid and cellobiosylamine derivative. Cellulose, 12(2), 209-219.

Karakawa, M., Mikawa, Y., Kamitakahara, H., \& Nakatsubo, F. (2002). Preparations of regioselectively methylated cellulose acetates and their H-1 and C-13 NMR spectroscopic analyses. Journal of Polymer Science Part A-Polymer Chemistry, 40(23), 4167-4179.

Kim, S., Stannett, V. T., \& Gilbert, R. D. (1973). A new class of biodegradable polymers. Journal of Polymer Science Polymer Letters Edition, 11, 731-735.

Kim, S., Stannett, V. T., \& Gilbert, R. D. (1976). Biodegradable Cellulose Block Copolymers. Journal of Macromolecular Science. Pt. A, Chemistry, A10(4), 671-679.

Kolb, H. C., Finn, M. G., \& Sharpless, K. B. (2001). Click chemistry: diverse chemical function from a few good reactions. Angew. Chem., Int. Ed., 40, 2004-2021. 
Lepage, M. L., Schneider, J. P., Bodlenner, A., \& Compain, P. (2015). Toward a Molecular Lego Approach for the Diversity-Oriented Synthesis of Cyclodextrin Analogues Designed as Scaffolds for Multivalent Systems. J. Org. Chem., 80, 10719-10733.

Meldal, M. (2008). Polymer "clicking" by CuAAC reactions. Macromol. Rapid Commun., 29, 1016-1051.

Nakagawa, A., Fenn, D., Koschella, A., Heinze, T., \& Kamitakahara, H. (2011a). Physical Properties of Diblock Methylcellulose Derivatives with Regioselective Functionalization Patterns: First Direct Evidence that a Sequence of 2,3,6-Tri-O-methyl-glucopyranosyl Units Causes Thermoreversible Gelation of Methylcellulose. Journal of Polymer Science Part B-Polymer Physics, 49(21), 1539-1546.

Nakagawa, A., Fenn, D., Koschella, A., Heinze, T., \& Kamitakahara, H. (2011b). Synthesis of Diblock Methylcellulose Derivatives with Regioselective Functionalization Patterns. Journal of Polymer Science Part A-Polymer Chemistry, 49(23), 4964-4976.

Nakagawa, A., Ishizu, C., Sarbova, V., Koschella, A., Takano, T., Heinze, T., \& Kamitakahara, H. (2012). 2-O-Methyl- and 3,6-Di-O-methyl-cellulose from Natural Cellulose: Synthesis and Structure Characterization. Biomacromolecules, 13(9), 2760-2768.

Nakagawa, A., Kamitakahara, H., \& Takano, T. (2011). Synthesis of blockwise alkylated (1-->4) linked trisaccharides as surfactants: influence of configuration of anomeric position on their surface activities. Carbohydr Res, 346(13), 1671-1683.

Nakagawa, A., Kamitakahara, H., \& Takano, T. (2012). Synthesis and thermoreversible gelation of diblock methylcellulose analogues via Huisgen 1,3-dipolar cycloaddition. Cellulose, 19(4), 1315-1326.

Nakagawa, A., Steiniger, F., Richter, W., Koschella, A., Heinze, T., \& Kamitakahara, H. (2012). Thermoresponsive Hydrogel of Diblock Methylcellulose: Formation of Ribbonlike Supramolecular Nanostructures by Self-Assembly. Langmuir, 28(34), 12609-12618.

Nakatsubo, F., Kamitakahara, H., \& Hori, M. (1996). Cationic ring-opening polymerization of 3,6-di-O-benzyl- $\alpha$-D-glucose 1,2,4-orthopivalate and the first chemical synthesis of cellulose. Journal of the American Chemical Society, 118(7), 1677-1681.

Pohjola, L., \& Eklund, V. (1977). Polyurethane block copolymers from cellulose acetate. Paperi ja Puu, 3, 117-120.

Savage, A. B. (1957). Temperature-Viscosity Relationships for Water-Soluble Cellulose Ethers. Industrial and Engineering Chemistry, 49, 99.

Steinmann, H. W. (1968). Novel cellulosics and segmented copolymers. (p. 17 pp.): Celanese Corp. .

Steinmann, H. W. (1970). Elastomeric fibers from cellulose acetate. Polym. Prepr., Am. Chem. Soc. Div. Polym. Chem., 11(1), 285-290. 
459 Figure Captions

460 Scheme 1. Synthesis of tri-O-methyl cellulosyl azide (2) and propargyl tri-O-methyl celluloside (3) 461 from tri-O-methyl cellulose (1).

462 Figure 1. MALDI-TOF MS spectra of $(\mathrm{A})$ tri-O-methyl cellulose (1) $\left(D P_{\mathrm{n}}=322\right)$, (B) tri-O-methyl 463 cellulosyl azide $(2)\left(D P_{n}=27.5\right)$, and $(C)$ propargyl tri-O-methyl celluloside $(3)\left(D P_{\mathrm{n}}=13.2\right)$.

464 Figure 2. ${ }^{1} \mathrm{H}-\mathrm{NMR}$ spectra of $(\mathrm{A})$ tri-O-methyl cellulose (1) $\left(D P_{\mathrm{n}}=322\right)$, (B) tri-O-methyl 465 cellulosyl azide (2) $\left(D P_{\mathrm{n}}=27.5\right)$, and $(\mathrm{C})$ propargyl tri-O-methyl celluloside (3) $\left(D P_{\mathrm{n}}=71.2\right)$.

466 Figure 3. ${ }^{13} \mathrm{C}-\mathrm{NMR}$ spectra of (A) tri-O-methyl cellulose (1) $\left(D P_{\mathrm{n}}=322\right)$, (B) 467 tri-O-methylcellulosyl azide (2) $\left(D P_{\mathrm{n}}=27.5\right)$, and $(\mathrm{C})$ propargyl tri-O-methyl celluloside (3) $\left(D P_{\mathrm{n}}\right.$ $468=71.2)$.

470 Table 1. Azido end-functionalization of tri-O-methyl cellulose (1).

471 Table 2. Propargyl end-functionalization of tri-O-methyl cellulose (1). 